Marcel Guéridon*

\title{
Stand und Herausforderungen der Forschung zum Anstaltsklima im deutschen Justizvollzug - zwischen vielversprechenden Anfängen und drohender Verzettelung
} Status Quo and Challenges Concerning Prison Climate Research in the German Prison System Between Promising Beginnings and Risk of Fragmentation

https://doi.org/10.1515/mks-2020-2049

Zusammenfassung: Das Anstaltsklima ist in den letzten Jahren zunehmend Thema der Forschung auch im deutschen Justizvollzug geworden. Diese positive Entwicklung leidet jedoch bisher unter einer hohen Fragmentierung der verschiedenen Projekte und einer Reihe theoretischer, forschungspraktischer und methodischer Probleme. Diese reichen von der Frage der Definition über mangelnde Berücksichtigung von Mehrebenenstrukturen und der Beschränkung auf querschnittliche Forschungsdesigns bis hin zu Problemen der Objektivität angesichts unterschiedlicher Perspektiven. Der vorliegende Beitrag gibt eine kurze Literaturübersicht über Forschungsprojekte jüngeren Datums zum Klima im Justizvollzug in Deutschland anhand von Veröffentlichungen ab der Jahrtausendwende. Im Anschluss werden methodische und konzeptuelle Herausforderungen hervorgehoben, die mit der Erhebung und Erforschung des Klimas im Justizvollzug in Theorie und Praxis einhergehen. Aus diesen werden eine Reihe von Empfehlungen für die Klimaforschung im Justizvollzug abgeleitet und diskutiert.

Schlüsselwörter: Anstaltsklima, Justizvollzug, Strafvollzugsforschung, Literaturübersicht

Abstract: Prison climate has become an increasingly investigated subject in German prison research. At this time,

*Kontaktperson: M.Sc. Marcel Guéridon, Kriminologischer Dienst im Bildungsinstitut des niedersächsischen Justizvollzuges, Fuhsestraße 30, 29221 Celle,

E-Mail: marcel.gueridon@justiz.niedersachsen.de this desirable development suffers from serious fragmentation and a number of theoretical, practical and methodological problems. Among these are questions of definition, a lack of attention to multilevel structures, restriction to cross-sectional research designs and problems of objectivity. This contribution gives a short literature review of recent German research projects concerning prison climate based on publications since 2000. In addition, some methodological and conceptual challenges regarding climate research in prisons are highlighted. Recommendations for future research are inferred and discussed.

Keywords: Prison climate, German prison research, literature review

\section{Einleitung}

Das Klima in Justizvollzugsanstalten kann aus ganz verschiedenen Gründen als ein wichtiger Faktor für das Gelingen oder Scheitern einer Justizvollzugseinrichtung gesehen werden (Guéridon \& Suhling 2018, 251). Nachdem das Thema über viele Jahre hinweg eher selten Gegenstand der Strafvollzugsforschung war, ist es in letzter Zeit sowohl international als auch in Deutschland vermehrt in den Fokus gerückt. Diese positive Entwicklung leidet jedoch bisher wie die Forschung zum Klima im Justizvollzug insgesamt unter einer hohen Fragmentierung: Verschiedene Arbeitsgruppen arbeiten mit verschiedenen Instrumenten (EssenCES, PGCI, MQPL) in verschiedenen Einrichtungen und Bundesländern an verschiedenen Facetten (Klima zwi- 
schen Bediensteten und Inhaftierten, Klima zwischen Bediensteten usw.), auf verschiedenen Ebenen (Gruppenklima, Abteilungsklima, Anstaltsklima) und an verschiedenen Themen bzw. abhängigen Variablen (Gesundheit, Zufriedenheit, Behandlungswirksamkeit, Gewalt) ohne systematische Verknüpfung dieser mit einem Schwerpunkt bei den traditionell eher »behandlungsorientierten « Einrichtungen (Sozialtherapie, Jugendanstalten).

Ein wichtiger Grund dafür ist das komplexe Konstrukt »Klima« selbst, welches mit einer Reihe von Herausforderungen auf konzeptueller, forschungstheoretischer und forschungspraktischer Seite einhergeht. Es wird oft als »Persönlichkeit« der Einrichtung (Moos 1997) oder als »das von den beteiligten Personen erlebte Gesamt der materiellen, sozialen und emotionalen Gegebenheiten einer Institution« (Schalast \& Groenewald 2009, 329) verstanden, aber im Detail stellt schon die Definition angesichts verschiedener Schwerpunkte und Begriffe (soziales Klima, Institutionsklima, Organisationsklima, Behandlungsklima, soziale Atmosphäre usw.) eine Herausforderung dar (Guéridon \& Suhling 2018).

Dieser Beitrag gibt eine kurze Literaturübersicht aktueller Forschungsprojekte zum »Klima« von Justizvollzugseinrichtungen in Deutschland anhand von Veröffentlichungen ab der Jahrtausendwende. Auf dieser Grundlage werden einige methodische und konzeptuelle Herausforderungen hervorgehoben, die mit der Erhebung und Erforschung des Klimas im Justizvollzug in Theorie und Praxis einhergehen. Aus diesen werden abschließend eine Reihe von Empfehlungen für die Klimaforschung im Justizvollzug abgeleitet.

\section{Der Stand der Forschung zum Klima im Justizvollzug}

Das Klima in Einrichtungen des Justizvollzugs und damit verknüpfte Themen wurden auch schon vor der Jahrtausendwende in Deutschland vereinzelt beforscht und diskutiert (siehe z. B. Braune, Klapprott, Linz, Lösel \& Runkel 1983; Dahle \& Steller 1990; Lösel, Köferl \& Weber 1987 [Kapitel 8]; Ortmann 2002). Dennoch kann spätestens ab Mitte der 2000er Jahre eine intensivere Auseinandersetzung festgehalten werden, die in den letzten Jahren noch einmal zugenommen hat. Tabelle 1 (S. $274 \mathrm{f}$.) zeigt die auffindbaren Projekte mit jeweils wichtigen Publikationen. Grundlage war vor allem die über die Jahre gesammelte Literatur im Rahmen weiterer Veröffentlichungen (Guéridon \& Suhling 2018) und eines eigenen Projektes (Guéridon 2019) sowie
- Recherchen über einschlägige Datenbanken und Suchmaschinen (Criminal Justice Abstracts; KrimDok; Google Scholar), im Wesentlichen anhand der Begriffe »Klima«/»prison climate« sowie »Justizvollzug« bzw. »Strafvollzug«;

- die Durchsicht deutschsprachiger kriminologischer Zeitschriften ab dem Jahrgang 2000 (Forum Strafvollzug; Bewährungshilfe; Monatsschrift für Kriminologie und Strafrechtsreform; Forensische Psychiatrie, Psychologie, Kriminologie; Recht \& Psychiatrie; Neue Kriminalpolitik; Zeitschrift für Jugendkriminalrecht und Jugendhilfe);

- die Durchsicht aller Vorgänge zu externer Forschung im niedersächsischen Justizvollzug; ${ }^{1}$

- Durchsicht der Veröffentlichungslisten relevanter Forschungseinrichtungen und Lehrstühle sowie gegebenenfalls gezielte Abfragen aktueller Ergebnisse bei Wissenschaftlern und Wissenschaftlerinnen, die in der Strafvollzugsforschung aktiv sind und/oder $\mathrm{zu}$ dem Thema veröffentlicht haben.

Angesichts der Zahl der aktuellen Veröffentlichungen kann durchaus von einer "Mode» gesprochen werden. Diese Bezeichnung sollte nicht pejorativ verstanden werden. Vielmehr lässt sich argumentieren, dass sogar noch mehr Klimaforschung nötig ist (Guéridon \& Suhling 2018).

Da es sich bei der vorliegenden Arbeit um eine problemzentrierte Übersicht handelt, wird statt einer streng chronologischen oder inhaltlichen Aufarbeitung der Literatur im Einzelnen eine Aufstellung nach bestimmten auffälligen Beobachtungen (z. B. bezüglich Zielgruppe, Forschungsansatz usw.) in Form von sieben Thesen verfolgt. Zudem wird der Bereich des therapeutischen Gruppenklimas in spezifischen Behandlungs- und Fördermaßnahmen ausgeklammert. Dieser würde eine gesonderte Auswertung erfordern. Wo es sich anbietet (z.B. zur Darstellung alternativer Ansätze oder wichtiger Bezüge), werden auch internationale Beiträge herangezogen. Der Schwerpunkt liegt jedoch auf der Klimaforschung im deutschen Justizvollzug. Durchgehend berücksichtigt werden aktuelle Ergebnisse einer Studie mit dem Essen Climate Evaluation Schema (EssenCES) aus der Schweiz (Isenhardt, Mangold \& Hostettler 2020). Auf eine weitergehende Berücksichtigung der umfangreichen aktuellen Ergebnisse aus den

1 Auf eine Abfrage bei den Kriminologischen Diensten der anderen Bundesländer wurde verzichtet. Da es sich in diesem Bereich gegebenenfalls vor allem um "graue« Literatur zu überwiegend eher kleineren Projekte und Qualifikationsarbeiten handeln wird, dürfte diese nicht im Kern die hier formulierten Thesen und Befunde in Frage stellen können. 
Niederlanden (Beijersbergen, Dirkzwager, van der Laan \& Nieuwbeerta 2016; Bosma, van Ginneken, Sentse \& Palmen 2019/van Ginneken, Palmen, Bosma, Nieuwbeerta \& Berghuis 2018/van Ginneken, Palmen, Bosma \& Sentse 2019²) wird dagegen verzichtet, da die verwendeten Instrumente (z. B. Prison Climate Questionnaire, PCQ) unseres Wissens bislang nicht in Deutschland verwendet wurden. ${ }^{3}$ Wegen ihrer Besonderheiten werden einige Veröffentlichungen aus dem Bereich des Maßregelvollzugs berücksichtigt, wobei keine weitergehende Literaturrecherche $\mathrm{zu}$ diesem Feld durchgeführt wurde. Für eine umfangreichere Darstellung auch des internationalen Forschungsstandes zum Klima im Justizvollzug siehe Guéridon \& Suhling (2018). Neben den hier genauer beschriebenen empirischen Arbeiten lassen sich auch eine Reihe von theoretischen Aufsätzen und Überblicksarbeiten finden (z. B. Drenkhahn 2011; Guéridon \& Suhling 2018; Obergfell-Fuchs 2016; van den Boogaart 2014).

\subsection{Die Klimaforschung im deutschen Justizvollzug beschränkt sich fast ausschließlich auf behandlungsorientierte Einrichtungen}

Sozialtherapeutische Einrichtungen sind häufig Gegenstand der Klimaforschung im deutschen Justizvollzug. Dies ist insoweit wenig überraschend, da das therapeutische Klima ja gerade ein wesentlicher Faktor der »Sozialtherapie« sein soll (van den Boogaart 2014) und es eine Reihe von großangelegten Evaluationsprojekten gibt, die auch das Klima berücksichtigen (Guéridon 2019; Klein, Schmucker \& Lösel 2015; Ortmann 2002; Sauter et al. 2019/Stasch et al. 2018; Seifert \& Thyrolf 2010; Suhling, Köhler \& Bernardi 2009; Wößner \& Schwedler 2014). Der Vergleich mit Einrichtungen des Regelvollzugs, der Sicherungsverwahrung und des Maßregelvollzugs ist dabei immer wieder Thema (Ortmann 2002; Sauter et al. 2019;

2 In diesem Beitrag wird in der Zitation durch ein »/ « angedeutet, dass die Veröffentlichungen dem gleichen Projekt zugeordnet werden können. Gegebenenfalls werden die Veröffentlichungen in der Zitation zusammengezogen, auch wenn die Auflistung dadurch nicht alphabetisch ist.

3 Eine in vielen Punkten identische Diskussion wurde zwischenzeitlich (nach Einreichung dieses Beitrags) von van Ginneken \& Nieuwbeerta (2020) für die internationale Forschung zum Klima im Justizvollzug veröffentlicht. Dort werden allerdings deutschsprachige Veröffentlichungen nicht berücksichtigt, weshalb der vorliegende Beitrag hoffentlich dennoch einen Mehrwert und eine in Teilen ergänzende Perspektive bietet.
Schalast \& Groenewald 2009; Suhling, Köhler \& Bernardi 2009), oft werden aber auch nur sozialtherapeutische Einrichtungen betrachtet (Guéridon 2019/Guéridon \& Strecker, im Druck; Seifert \& Thyrolf 2010; Wößner \& Schwedler 2014). Meist werden in den Untersuchungen zur Sozialtherapie männliche erwachsene Inhaftierte befragt, seltener (auch) Jugendliche (Guéridon 2019; Sauter et al. 2019) und Frauen (Guéridon 2019).

Jugendliche und heranwachsende Inhaftierte sind jenseits der Forschung zur Sozialtherapie allerdings häufig Thema der Klimaforschung (Adler 2015; Boxberg et al. 2016/Neubacher \& Boxberg 2018; Heynen et al. 2014a/ Heynen et al. 2014b/Heynen et al. 2014c/Mohr et al. 2019; Prätor \& Suhling, in Vorb.; Schalast \& Groenewald 2009/ Schalast \& Laan 2017). Auch in den großangelegten Erhebungen (Baier et al. 2014/Klatt et al. 2017; Isenhardt, Mangold \& Hostettler 2020) werden in der Regel Daten zu Jugendlichen/Heranwachsenden sowie Frauen erhoben, allerdings nicht gesondert ausgewiesen.

Schließlich ist auch die Sicherungsverwahrung in den letzten Jahren in den Fokus der Klimaforschung gekommen (Sauter et al. 2019/Stasch, Sauter \& Dahle 2017/ Stasch et al. 2018). Auch im Maßregelvollzug ist das Klima ein Thema (Pniewski 2019/Pniewski, Elsner \& König 2019), gerade durch die langjährige Tätigkeit von Norbert Schalast (Schalast 2019; Schalast \& Redies 2005).

Insgesamt wird damit deutlich, dass sich die Klimaforschung abgesehen von einigen Großprojekten (Baier et al. 2014/Klatt et al. 2017; Boxberg et al. 2014/Neubacher \& Boxberg 2018; Isenhardt, Mangold \& Hostettler 2020) auf eher behandlungsorientierte "Sondervollzüge« konzentriert. Obwohl in diesen Bereichen das Thema Klima auch konzeptuell eine besondere Rolle hat, kann ein Mangel an Forschung im Regelvollzug/«Normalvollzug« festgehalten werden.

\subsection{Bedienstete und Inhaftierte sind gleichermaßen im Fokus, häufig aber nicht gemeinsam}

In der Literatur dominiert die Perspektive der inhaftierten Personen. Vereinzelt wurden allerdings auch Untersuchungen nur mit Bediensteten durchgeführt (Lehmann \& Greve 2006; Girisch 2014), einige aktuelle Projekte beziehen auch beide Perspektiven ein (Adler 2015; Guéridon 2019/Guéridon \& Strecker, im Druck/Strecker 2018; Sauter et al. 2019; Schalast \& Groenewald 2009/Schalast \& Laan 2017). Dies mag auch daran liegen, dass derzeit lediglich das EssenCES (Schalast \& Tonkin 2016) ohne größere Herausforderungen gleichermaßen für Inhaftierte und Be- 
dienstete verwendet werden kann, während beispielsweise die SQL (»Staff Quality of Life«) als Pendant zur MQPL (»Measuring the Quality of Prison Life«, Liebling, Hulley \& Crewe 2011) nicht als validierte deutschsprachige Version zur Verfügung steht. Die »Celler Klima Skalen« (CKS, Suhling, Köhler \& Bernardi 2009) wurden vom Kriminologischen Dienst Niedersachsen mit geringen Anpassungen auch in einer Variante für die Bediensteten getestet (Guéridon 2019), allerdings handelt es sich hierbei im Vergleich zum EssenCES um ein deutlich weniger erprobtes Instrument. Der Schwerpunkt auf die Perspektive der Inhaftierten lässt sich zudem aber auch vermutlich mit der nächsten Beobachtung erklären: Oft steht individueller Behandlungserfolg bzw. individuelles Fehlverhalten der Inhaftierten im Fokus. Für entsprechende Fragestellungen ist die Perspektive der Inhaftierten leichter zu erheben und mit weiteren Daten zu kombinieren als die Perspektive der Bediensteten, die noch einmal erheblichen zusätzlichen Aufwand darstellt. Im Zweifelsfall liegt es daher durchaus nahe, eher oder nur die Perspektive der Inhaftierten abzubilden. Problematisch bleibt aber die Verallgemeinerung (vgl. Guéridon \& Strecker, im Druck).

\subsection{Klima wird in der Regel als unabhängige Variable im Kontext von Fehlverhalten und der Behandlung untersucht}

Neben der Orientierung auf therapeutische Einrichtungen lassen sich auch inhaltlich bestimmte Schwerpunkte feststellen. Die meisten Studien zum Klima in Justizvollzugseinrichtungen in Deutschland betrachten die Wirkung des Klimas (Klima als unabhängige Variable). Oft geht es dabei um den Zusammenhang von Klima und Fehlverhalten/Gewalt (Boxberg et al. 2014/Neubacher \& Boxberg 2018; Baier et al. 2014/Klatt et al. 2017; Prätor \& Suhling, in Vorb.) oder um den Zusammenhang mit Behandlungserfolg bzw. -teilnahme (Ortmann 2002; Stasch et al. 2018; Schalast 2019; Suhling, Köhler \& Bernardi 2009; Wößner \& Schwedler 2014). Ein zweiter Schwerpunkt betrifft die Beschreibung und Dokumentation möglicher Veränderungen des Klimas (Adler 2015; Guéridon 2019; Heynen et al. 2014a/Heynen et al. 2014b/Heynen et al. 2014b/Mohr et al. 2019; Klein, Schmucker \& Lösel 2015; Pniewski 2019/Pniewski, Elsner \& König 2019; Schalast \& Groenwald 2009/Schalast \& Laan 2017; Seifert \& Thyrolf 2010; Stasch et al. 2017). Unter diesem Punkt lassen sich auch die regelmäßigen Vergleiche zwischen bestimmten Einrichtungen und Einrichtungsformen fassen, die in den meisten Fällen behandlungsorientierte Einrichtungen dem Regelvollzug gegenüberstellen oder seltener eine auf die Einrichtung bezogene zeitliche
Perspektive beinhalten (Stasch et al. 2017). Nur in Ausnahmefällen wird das Klima dagegen als echte abhängige Variable untersucht (Guéridon 2019; Sauter et al. 2019), was oft an der geringen Zahl der betrachteten Einrichtungen liegen mag.

Bei den Bediensteten liegt der Schwerpunkt oft eher im Bereich der Arbeitszufriedenheit und der Gesundheit (Girisch 2014; Lehmann \& Greve 2006; Sauter et al. 2019) und fokussiert damit eher das Betriebs- oder Teamklima.

\subsection{Das Klima wird - zumindest auf Abteilungsebene - meist nur im Querschnitt erhoben}

Viele Aufsätze beschreiben einmalige Messungen des Klimas (Adler 2015; Baier et al. 2014/Klatt et al. 2017; Guéridon \& Strecker, im Druck/Strecker 2018; Girisch 2014; Lehmann \& Greve 2006; Schalast \& Groenewald 2009/ Schalast \& Laan 2017; Suhling, Köhler \& Bernardi 2009).

Einige Studien verfolgen das Klima allerdings auch über die Zeit. Dabei können zwei Varianten unterschieden werden: Die individuelle Wiederholungsmessung (z.B. Beginn und Abschluss einer Behandlungsmaßnahme) und die Wiederholungsmessung auf Einrichtungsebene. Die individuelle Wiederholungsmessung ist durchaus verbreitet (Klein, Schmucker \& Lösel 2015; Ortmann 2002; Seifert \& Thyrolf 2010; Wößner \& Schwedler 2014), die Wiederholungsmessung auf Abteilungsebene (Guéridon 2019; Pniewski 2019/Pniewski, Elsner \& König 2019; Mohr et al. 2019) ist dagegen seltener. Dabei können noch einmal zwei Sonderformen unterschieden werden: die Auswertung nach Aufnahmejahrgängen (interindividuelle Messung, z.B. Seifert \& Thyrolf 2010) und die retrospektive Abfrage (intraindividuelle Messung, ebenfalls Seifert \& Thyrolf 2010; Stasch et al. 2017).

\subsection{Das Klima wird nicht einheitlich gemessen}

Obwohl die Klimadefinitionen oft vergleichbar sind, werden in der Strafvollzugsforschung eine ganze Reihe von Instrumenten verwendet. Am häufigsten wird in Deutschland das EssenCES genutzt (Pniewski 2019/Pniewski, Elsner \& König 2019; Sauter et al. 2019/Stasch et al. 2018/ Stasch et al. 2017; Schalast \& Groenewald 2009/Schalast \& Laan 2017). Zum Teil wird das EssenCES mit weiteren Verfahren kombiniert, etwa den CKS (Guéridon 2019; Prätor \& Suhling, in Vorb.) oder der gekürzten Correctional Institutions Environment Scale (S-CIES, Adler 2015; Klein, 
Schmucker \& Lösel 2015). Verwendet werden zudem die von Ortmann (2002) konstruierten Skalen (Adler 2015; Klein, Schmucker \& Lösel 2015; Wößner \& Schwedler 2014), Inhalte aus der MQPL (Baier et al. 2014; Klatt et al. 2017), dem erst kürzlich entwickelten Prison Group Climate Instrument (PGCI, Heynen et al. 2014a/Heynen et al. 2014b/Mohr et al. 2019) und in vielen Fällen auch (globale) ad-hoc konstruierte Fragen (Girisch 2014; Lehmann \& Greve 2006; Seifert \& Thyrolf 2010; ergänzend auch bei Adler 2015; Guéridon 2019; Klein, Schmucker \& Lösel 2015).

Neben der Befragung als typischem Zugang ist - alternativ oder ergänzend - auch ein auf Beobachtung bzw. Begutachtung basierender Ansatz denkbar. Inwieweit aber tatsächlich über (teilnehmende) Beobachtung und beispielsweise Gebäuderundgänge eine Art kontextpsychologische Begutachtung des Klimas möglich ist, kann nur schlecht eingeschätzt werden. Man kann vermuten, dass dieses Vorgehen in der Praxis durchaus von Relevanz ist, ohne dabei systematisch wissenschaftlichen Kriterien zu entsprechen. Systematische Ansätze sind zumindest bisher nicht in der deutschsprachigen Literatur zu finden. Am ehesten integriert der MQPL+-Ansatz von Liebling (Liebling, Hulley \& Crewe 2011), der weit über die simple Anwendung des MQPL/SQL hinausgeht, auch solche Überlegungen. Auch vorwissenschaftliche Bewertungen des Klimas durch externe Personen (z.B. im Rahmen von Aufsichtsbesuchen o.Ä.) erbringen zumindest anekdotisch Belege für konsistente Einschätzungen des Klimas. Bislang sind aus der deutschen Strafvollzugsforschung aber keine Versuche bekannt, diesen Ansatz auszubauen oder mit Befragungen der Inhaftierten und Bediensteten abzugleichen.

\subsection{Der Rücklauf in der Klimaforschung ist gut, besonders bei den Inhaftierten}

Für die allermeisten Projekte konnten Rücklaufquoten ermittelt werden. Dabei zeigen sich zwei Auffälligkeiten: Zum einen weisen Projekte, die ausschließlich das Klima zum Thema haben (Guéridon \& Strecker, im Druck; Heynen et al. 2014a/Heynen et al. 2014b/Mohr et al. 2019; Schalast \& Groenewald 2009/Schalast \& Laan 2017; Suhling, Köhler \& Bernardi 2009), überwiegend Rücklaufquoten von über $80 \%$ bei den Inhaftierten auf. Ausnahmen sind hierbei die Arbeiten von Adler (2015: 49,6\%) und die Totalerhebung von Isenhardt, Mangold \& Hostettler (2020) mit immer noch $70 \%$ Rücklauf. In Untersuchungen, in denen das Klima nur ein Teilaspekt ist, fällt die Quote geringer aus (Baier et al. 2014/Klatt et al. 2017: $50 \%$; Boxberg et al. 2016: 62-75\%). Auch hier gibt es allerdings positive Beispiele (Ortmann 2002; Seifert \& Thyrolf 2010; Sauter et al. 2019/Stasch et al. 2017/Stasch et al. 2018; Prätor \& Suhling, in Vorb.). In jedem Fall scheint Klimaforschung im Justizvollzug möglich, eine Rücklaufquote von mindestens $50 \%$ ist sicher zu erwarten, eigentlich sogar deutlich höhere Quoten.

Bei den Bediensteten fallen die Rücklaufquoten insgesamt geringer aus. Diese reichen von ca. 50 \% (Girisch 2015; Lehmann \& Greve 2006; Sauter et al. 2019) bis maximal $70 \%$ (Guéridon \& Strecker, im Druck). In der Schweizer Totalerhebung (Isenhardt, Mangold \& Hostettler 2020) liegt die Quote demgegenüber nur bei $37 \%$. Ob diese Unterschiede an unterschiedlichem Anreiz, fehlender Zeit oder fehlendem Interesse liegt oder ganz andere Ursachen hat, ist bisher nur selten diskutiert worden. Der aus einer konflikttheoretischen Perspektive heraus formulierte Beitrag von Pniewski \& Walsh (2018) zur Evaluations- und Implementationsforschung in der Praxis stellt hier eine wichtige Ausnahme dar. Konkret auf das Klima bezogen mangelt es allerdings an »Meta«-Forschung zu Bedingungen und Ursachen (erfolgreicher) Implementation auch bei Bediensteten. Der Eindruck aus der eigenen Forschung und der Diskussion mit Kolleginnen und Kollegen ist zumindest, dass viel vom Engagement bestimmter Personen vor Ort abhängt (Abteilungsleitung oder Ansprechpartner). Es scheint aber ebenfalls ratsam, a) Befragungen zum Klima nicht übermäßig mit anderen Inhalten zu verbinden (Ökonomie) und b) eine geeignete Erhebungsstrategie zu nutzen. In der eigenen (Online-)Erhebung (Guéridon 2019) konnten durch insgesamt bis zu zwei Erinnerungen noch einmal deutlich mehr Teilnehmerinnen und Teilnehmer gewonnen werden. ${ }^{4}$

\subsection{Es gibt graue Literatur zum Klima in Justizvollzugseinrichtungen}

Aus der privilegierten Situation heraus, die Strafvollzugsforschung in Niedersachsen notwendigerweise genau zu kennen, lässt sich die Vermutung aufstellen, dass einige Ergebnisse zum Klima nicht oder nur eingeschränkt bekannt sind. Die Masterarbeiten von Strecker (2018) und Adler (2015) dürften eher selten, die Dissertation von Girisch (2014) vermutlich nicht breit bekannt sein. Zumin-

4 Die genaue Quote kann nicht bestimmt werden, da natürlich nicht bekannt ist, ob eine Person auch ohne Erinnerung noch an der Befragung teilgenommen hätte. Aus den Teilnahmedaten pro Einrichtung lässt sich jedoch trotzdem recht sicher ableiten, dass ca. ein Drittel erst nach erneuter Bitte/Erinnerung durch den Autor bzw. durch die jeweilige Abteilungsleitung an der Befragung teilgenommen hat (38 von 123 Personen). 


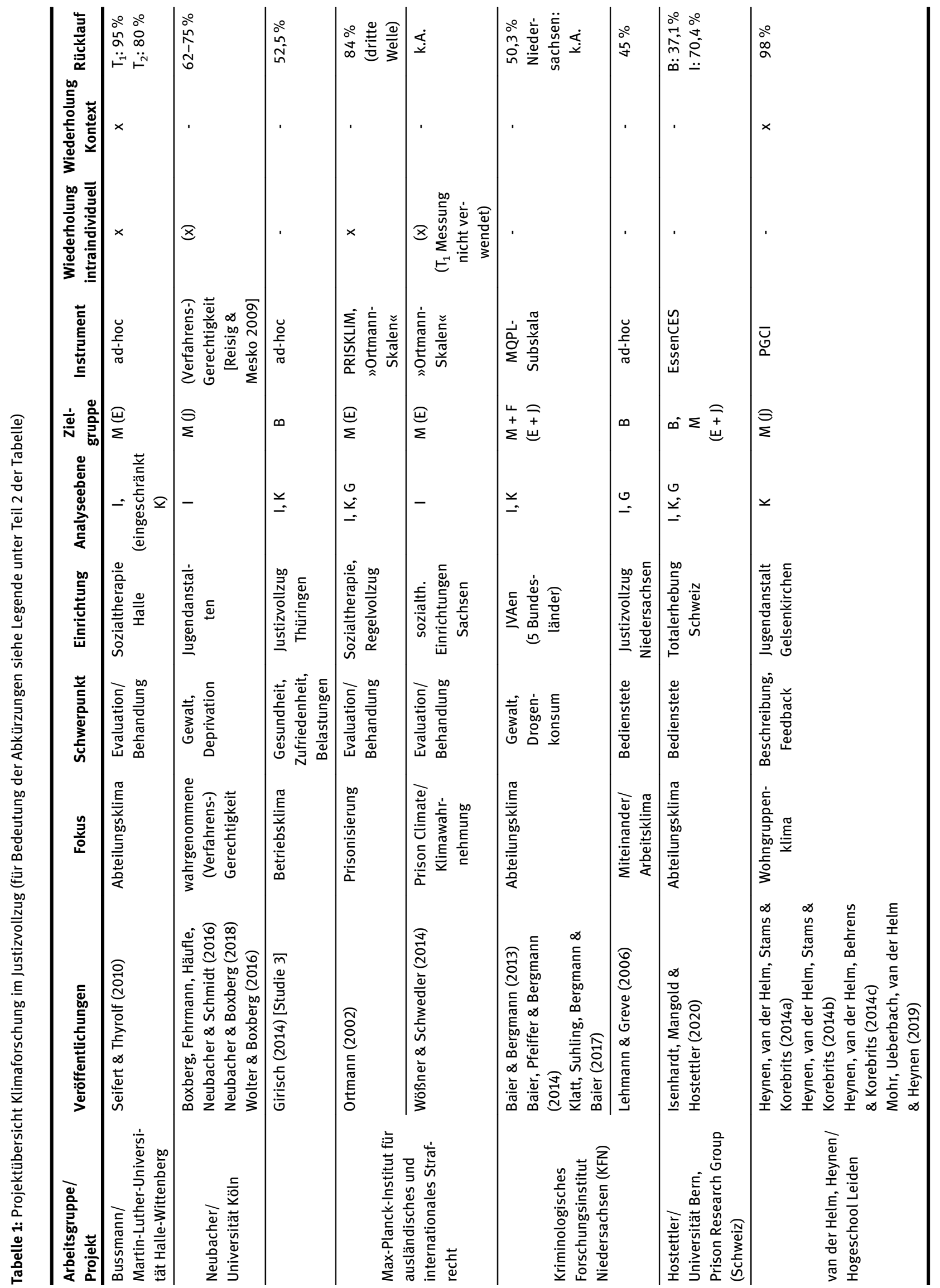




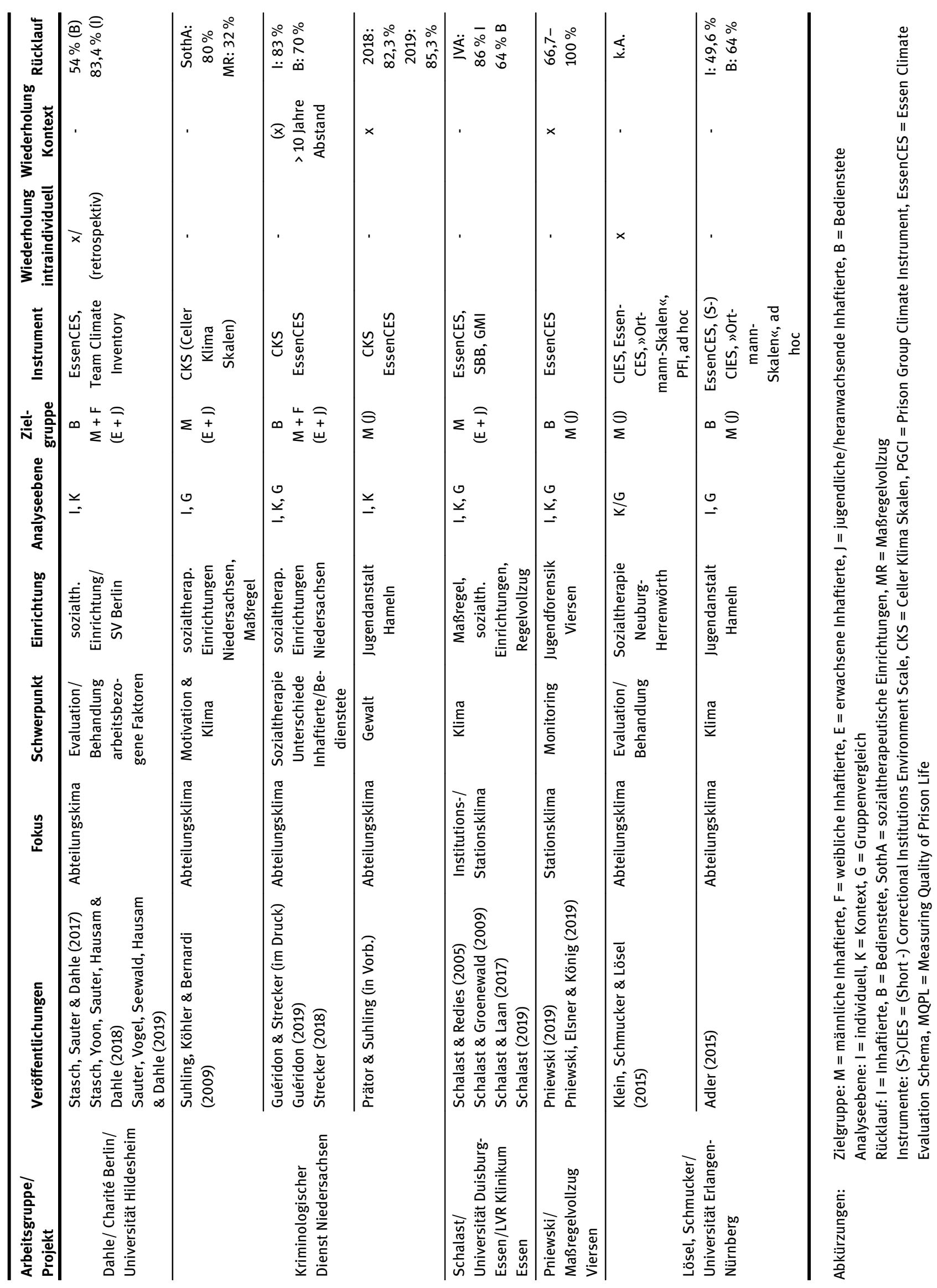


dest für Niedersachsen kann zudem festgehalten werden, dass einige Ergebnisse bisher nur intern kommuniziert und noch nicht veröffentlich wurden (Guéridon 2019; Prätor \& Suhling, in Vorb.). Ob und wie viel Forschung in ähnlichem Rahmen außerhalb von Niedersachsen stattfindet, kann auf Grundlage der eingeschränkten Literaturrecherche nicht eindeutig bestimmt werden. Insbesondere ist aber fraglich, ob und in welchem Umfang beispielsweise das EssenCES im Regelbetrieb (also vergleichbar zu Pniewski 2019/Pniewski, Elsner \& König 2019 oder Mohr et al. 2019) verwendet wird, ohne dass daraus wissenschaftliche Veröffentlichungen entstehen. ${ }^{5}$

\section{Herausforderungen, Lücken und Grenzen der Klimaforschung im Justizvollzug}

Aus der Übersicht der Forschungsstrategien und dem aktuellen Stand der Forschung (Guéridon \& Suhling 2018) lassen sich zumindest fünf Herausforderungen ableiten, mit denen sich die Klimaforschung im Justizvollzug (mehr) befassen sollte oder muss. In diesem Abschnitt werden diese Herausforderungen getrennt diskutiert, auch wenn sie vielfältige Überschneidungen aufweisen.

\subsection{Das Klimakonzept: Messen wir das gleiche Konstrukt?}

Es gibt eine Reihe von Definitionen für das Klima, die in der Literatur verbreitet sind, ohne dass sich die Forschenden bisher auf eine präzise Definition einigen konnten (siehe Guéridon \& Suhling 2018, 240 ff.). Dazu passt, dass die verschiedenen vorliegenden Fragebögen zur Messung des Klimas zwar häufig ähnliche, aber doch recht unterschiedliche Bereiche abdecken. Tabelle 2 gibt einen Überblick über die erfassten Dimensionen gebräuchlicher Verfahren in der deutschsprachigen Forschung zum Klima in Justizvollzugseinrichtungen (siehe van der Helm, Stams \& van der Laan [2011] für eine Gegenüberstellung englischsprachiger Instrumente). Neben dem Umfang der Skalen und der Breite erfasster Facetten unterscheidet sich auch die Perspektive auf Gruppen, Institutionen oder doch vor allem auf die Interaktion zwischen Gruppen. Angesichts der verschiedenen Ziele, Perspektiven und Facetten stellt sich schnell die

5 Entsprechende Arbeiten bzw. Hinweise können gern an den Autor übersandt werden.
Frage, ob eigentlich das gleiche Konzept betrachtet wird. Auch wenn sich auf den ersten Blick inhaltliche Überschneidungen zeigen, bleibt dies letztlich eine empirische Frage. Bisher gibt es jedoch nur wenige Versuche, die Konstruktvalidität der verwendeten Skalen zu untersuchen, wobei das EssenCES positiv heraussticht (Schalast \& Redies 2005; Schalast \& Tonkin 2016; Vries et al. 2018; für die englischsprachige Version: Tonkin et al. 2012). In einer eigenen Untersuchung zum Klima in sozialtherapeutischen Einrichtungen (228 inhaftierte Personen und 122 Bedienstete in 11 Abteilungen) zeigten sich ebenfalls sehr deutliche Korrelationen (z.B. $r=.88$ für die Skalen zur „Unterstützung durch Bedienstete“, $p>$.001) zwischen den EssenCES-Skalen und den CKS-Skalen auf Personenebene (Guéridon 2019).

Zugleich bleibt sowohl was das Konstrukt als auch die Wirkung des Klimas angeht bisher offen, ob die richtige Ebene der Betrachtung eigentlich das Klima (als globales Merkmal) oder eher Klimafacetten (also die konstituierenden Dimensionen des Klimas, z.B. Unterstützung durch Bedienstete) die relevante Analyseebene sind. Die Autoren des EssenCES-Manuals (Schalast \& Tonkin 2016) betonen den Vorrang der Klimafacetten, aber oft finden sich in der Literatur entweder direkt gemessene, globale Maße des Klimas (z. B. Seifert \& Thyrolf 2010) oder zumindest auch global-aggregierte Werte (z. B. über die Facetten des EssenCES hinweg, etwa bei Sauter et al. 2019). Zugleich sind globale, etwa mit Schulnoten vergleichbare Einschätzungen als Indikatoren für die Nutzer und Rezipienten in der Praxis vermutlich besser handhabbar als eine Übersicht sämtlicher Facetten des MQPL. Hier muss zumindest die Frage gestellt werden, ob die Klimaforschung sich (auch) als Teil eines formativen Prozesses sieht und insofern die Ergebnisse gut nutzbar sein sollen oder ob es vor allem um die ausführliche und differenzierte Messung geht. Hier muss kein Widerspruch entstehen, aber gerade die breite Akzeptanz des EssenCES lässt zumindest vermuten, dass ein kurzes, auf wenige Facetten begrenztes Instrument von der Praxis eher angenommen und intensiver genutzt wird.

Generell bleibt die Frage, ob das Klima als eine Art Faktor zweiter Ordnung betrachtet werden sollte und wie unabhängig die Klimafacetten und die Wirkung des Klimas bzw. der Klimafacetten voneinander sind. Dabei kann grundsätzlich zwischen einem kompensatorischen oder non-kompensatorischen Verhältnis unterschieden werden. Eine kompensatorische Wirkung würde bedeuten, dass das Klima (und entsprechende Wirkungen) auch insgesamt "gut" sein kann, wenn einzelne Klimafacetten schlecht eingeschätzt werden (gutes Klima trotz geringer Unterstützung durch Bedienstete), eine non-kompensatorische Wirkung meint dagegen, dass alle Klimafacetten positiv gewertet sein müssen, um von einem guten Klima 
Tabelle 2: Regelmäßig verwendete Skalen und Facetten (Auswahl)

\begin{tabular}{|c|c|c|c|c|c|c|}
\hline $\begin{array}{l}\text { Measuring Quality } \\
\text { of Prison Life } \\
\text { (MQPL) } \\
\text { (eigene Übersetzung) }\end{array}$ & $\begin{array}{c}\text { Staff Quality of } \\
\text { Life (SQL)* } \\
\text { (eigene Überset- } \\
\text { zung) }\end{array}$ & $\begin{array}{l}\text { Prison Group } \\
\text { Climate Instru- } \\
\text { ment (PGCI) } \\
\text { (Heynen et al. } \\
\text { 2014a-c) }\end{array}$ & $\begin{array}{l}\text { Ortmann-Skalen } \\
\text { (Ortmann 2002) }\end{array}$ & $\begin{array}{l}\text { Correctional Insti- } \\
\text { tutions Environ- } \\
\text { ment Scale (CIES) } \\
\text { (gekürzt) } \\
\text { (Dahle \& Steller } \\
\text { 1990) }\end{array}$ & $\begin{array}{c}\text { Essen Climate } \\
\text { Evaluation Schema } \\
\text { (EssenCES) } \\
\text { (Schalast \& } \\
\text { Tonkin 2016) }\end{array}$ & $\begin{array}{c}\text { Celler Klima } \\
\text { Skalen (CKS) } \\
\text { (Suhling, Köhler \& } \\
\text { Bernardi 2009; } \\
\text { Guéridon 2019) }\end{array}$ \\
\hline $\begin{array}{l}\text { Verhältnis zwischen } \\
\text { Inhaftierten und } \\
\text { Bediensteten } \\
\text { Humanität } \\
\text { „Anstand« (decency) } \\
\text { Unterstützung } \\
\text { bürokratische } \\
\text { Legitimität } \\
\text { Fairness } \\
\text { Respekt } \\
\text { Vertrauen } \\
\text { Sicherheit } \\
\text { Drogen und Ausbeu- } \\
\text { tung } \\
\text { Familienkontakte } \\
\text { persönliche Entwick- } \\
\text { lung } \\
\text { Autonomie } \\
\text { Stress } \\
\text {... }\end{array}$ & $\begin{array}{c}\text { Behandlung } \\
\text { durch Vorgesetzte } \\
\text { Einstellung zu } \\
\text { Vorgesetzten } \\
\text { Verhältnis zu } \\
\text { Kollegen } \\
\text { Engagement } \\
\text { Stress } \\
\text { Sicherheitprofes- } \\
\text { sionelle } \\
\text { Unterstützung } \\
\text {... }\end{array}$ & Atmosphäre & $\begin{array}{l}\text { Begrenzung der } \\
\text { Autonomie } \\
\text { "Klima in der } \\
\text { Anstalt» }\end{array}$ & $\begin{array}{l}\text { Offenheit der } \\
\text { Kommunikation } \\
\text { zwischen Inhaf- } \\
\text { tierten } \\
\text { Offenheit der } \\
\text { Kommunikation } \\
\text { zwischen Beam- } \\
\text { ten und } \\
\text { Inhaftierten } \\
\text { Disziplin und } \\
\text { Kontrolle }\end{array}$ & $\begin{array}{c}\text { Unterstützung } \\
\text { durch Bedienstete }\end{array}$ & $\begin{array}{l}\text { Unterstützung un- } \\
\text { ter Inhaftierten } \\
\text { Konflikte unter } \\
\text { Inhaftierten } \\
\text { Unterstützung } \\
\text { durch Bedienstete } \\
\text { Konflikte zwischen } \\
\text { Inhaftierten und } \\
\text { Bediensteten } \\
\text { Sicherheitserleben }\end{array}$ \\
\hline
\end{tabular}

* nur bei Bediensteten abgefragt

(und positiven Wirkungen) ausgehen zu können. Eine explizite Auseinandersetzung mit diesen Fragen findet sich in der Literatur kaum, lediglich in Ausnahmefällen (Heynen et al. 2014a/Heynen et al. 2014b/Heynen et al. 2014c) wird auf Grundlage korrelierter Klimafacetten für ein nonkompensatorisches Modell argumentiert. Möglicherweise lassen sich auch unabhängige Cluster von Klimafacetten finden - es scheinen so oder so noch vielen Frage offen.

Eine weitere negative Auswirkung der unterschiedlichen Konzepte und verwendeten Instrumente ist - auch mit Bezug auf den folgenden Abschnitt -, dass die Ergebnisse eigentlich nur absolut (in Bezug auf die Skalierung) und nicht relativ (im Vergleich zu anderen Einrichtungen) bewertet werden können. Zwar gibt es im EssenCES-Manual abteilungsbezogene Normen, diese beruhen aber »nur" auf 33 Einrichtungen, die zu einem wesentlichen Teil »therapeutisch" gestaltet sind und auch internationale Justizvollzugskontexte einbeziehen. Ob die englischsprachigen Ergebnisse im Rahmen der Anwendung der MQPL verwendet werden können, müsste zumindest diskutiert werden. ${ }^{6}$

6 Bislang gibt es allerdings noch gar keine akzeptierte und veröffentlichte deutsche Übersetzung.

\subsection{Klima, Klimawahrnehmung, Klimabewertung}

Eine zentrale, wenn auch nur selten explizit beschriebene Herausforderung besteht in der Frage nach der richtigen Analyseebene. In Bezug auf das Klima können immer mindestens zwei Ebenen unterschieden werden: die subjektive Klimawahrnehmung (das "psychological climate«, Parker et al. 2003) und das Klima als Merkmal eines überindividuellen sozialen Kontexts (Gruppe, Abteilung, Organisation). In der Literatur spiegeln sich diese Perspektiven insofern wider, als einzelne Studien lediglich Zusammenhänge auf individueller Ebene beschreiben (z.B. Baier \& Bergmann 2013; Wößner \& Schwedler 2014; Suhling et al. 2009), andere hingegen das Klima (auch) als Kontextmerkmal betrachten (Klatt et al. 2017; Schalast 2019). Da die Klimawahrnehmung üblicherweise über die Zustimmung $\mathrm{zu}$ bestimmten Aussagen gemessen wird, kann noch einmal zwischen Klimawahrnehmung und Klimabewertung (Antwortverhalten) unterschieden werden. Im Idealfall fallen Klima, Wahrnehmung und Bewertung (Messung) zusammen. Sobald aber vermutet wird, dass bestimmte (unbewusste) Antwortstrategien (z.B. Verzerrung in Richtung sozialer Erwünschtheit) eine Rolle im 
Antwortverhalten spielen, müssen die Ergebnisse zumindest entsprechend eingeordnet werden. Die Unterscheidung wird daher spätestens dann relevant, wenn Unterschiede zwischen Gruppen innerhalb von Einrichtungen thematisiert werden (vertiefend dazu Guéridon \& Strecker, im Druck; siehe auch den folgenden Abschnitt 3.3).

Für bestimmte Aspekte scheint die subjektive Perspektive ohne größere Einschränkung sinnvoll zu sein. Die wahrgenommene (Verfahrens-)Gerechtigkeit (Neubacher \& Boxberg 2018) etwa scheint auf individueller Ebene ganz richtig angesiedelt. Andere Fragestellungen wirken auf dieser Analyseebene wiederum nahezu trivial. Es dürfte beispielsweise wenig überraschen, dass ein subjektiv gut bewertetes Klima durch die Bediensteten mit einer höheren Arbeitszufriedenheit einhergeht. Eine entsprechende Untersuchung hat schon a priori nie die Chance, die zugrunde liegende These $\mathrm{zu}$ widerlegen. Im Falle eines fehlenden Zusammenhangs würde mit Sicherheit eher die Messung als die Theorie infrage gestellt werden. Eine solche Analyse zeigt daher in der Tat eher auf, dass die verwendeten Instrumente valide sind (vgl. Greve [2001] für eine analoge Argumentation in Bezug auf Intention und Verhalten auf Basis von Smedslund [1984]). Schalast (2016; 2019, 113) äußert ähnliche Bedenken bezogen auf den Zusammenhang von Stationsklima und positiven Therapieerfahrungen.

Häufiger wird allerdings das Klima zwischen bestimmten Typen von Kontexten verglichen (z. B. Sozialtherapie vs. Regelvollzug) und/oder das Klima einzelner Kontexte gegenübergestellt (einzelne Abteilungen im Vergleich und/oder einzelne Abteilungen über die Zeit). Intuitiv und umgangssprachlich ist diese Analyseebene im Falle des Justizvollzugs die eigentlich interessante. Im Rahmen der Klimaforschung ist es ja gerade die zentrale These, dass Unterschiede im Klima als Kontextmerkmal Effekte erzeugen. Insofern sollte dieser Kontext auch berücksichtigt werden. Eine solche Perspektive wirft natürlich direkt die Frage auf, was das Klima eigentlich ist und wie es denn genau kausale Wirkung entfalten soll (siehe Abschnitt 3.5). Hier spielt es aber keine entscheidende Rolle, ob es sich beim Klima um den Anteil an "shared perceptions « (Parker et al. 2003), ein emergentes Phänomen (Sauter et al. 2019), ein funktional beschreibbares Konstrukt oder nur ein Epiphänomen handelt. Wichtig ist erst einmal die pragmatische Frage, ob es a) um die Erklärung, Vorhersage und Veränderung des Verhaltens von Personen geht, die ein subjektives Urteil über das Klima äußern (Klimabewertung), b) deren tatsächliche Klimawahrnehmungen (die von der Bewertung abweichen können) oder ob es c) um die Frage geht, wie das Klima in dieser Abteilung tatsächlich ist, was daraus folgt und wie man dieses ver- ändern kann. Es ist eine gänzlich andere Frage, ob eine Person besonders viel Stress erlebt, wenn sie ihre Umwelt negativ bewertet oder ob eine mehr oder weniger objektiv negative Umwelt dazu führt, dass Personen in dieser Umwelt besonders viel Stress erleben.

Wie bereits dargestellt, finden sich in der Literatur beide Perspektiven. Je nach theoretischer Ausrichtung und Fragestellung haben eben auch beide Analyseebenen ihre Berechtigung. Problematisch ist vor allem die Vermischung beider Perspektiven: Aus einer Auswertung auf individueller Ebene lässt sich nicht ableiten, dass das Klima als Kontextmerkmal einen Effekt hat. Es scheint daher notwendig, auf eine klare sprachliche und auch theoretische Unterscheidung zwischen Klima, Klimawahrnehmung und Klimabewertung zu bestehen. Soll die individuelle Situation oder Lebensqualität untersucht werden, ist die Klimabewertung vermutlich eine angemessene Auswertungsebene. Dabei ist eine genaue Abgrenzung zwischen Klimakonstrukt und Kriterium wichtig, um nicht nur triviale oder gar logisch implizierte Zusammenhänge abzubilden. Sollen aber Effekte von Deprivation, therapeutischem Setting oder Organisationsgesundheit thematisiert werden, scheint die Perspektive auf das Klima als Kontextmerkmal unausweichlich.

Diese klare konzeptuelle Trennung ist allerdings nur eine Seite der Herausforderung. Unabhängig von der konkreten Definition des Konstruktes (siehe Abschnitt 3.1) sind "Klima» und die Klimafacetten als Merkmal einer Gruppe, einer Abteilung oder einer Einrichtung ebenso wenig direkt messbar wie die subjektive Klimawahrnehmung. Die typische Strategie zur Bestimmung des Klimas liegt entsprechend darin, einen mittleren Wert aus den subjektiven Bewertungen der beteiligten Personen zu bilden. Die Alternative einer »klinischen« Urteilsbildung durch externe Personen ist wie schon angedeutet noch am ehesten im Rahmen der umfangreichen Verwendung des MQPL+ (Liebling, Hulley \& Crewe 2011) verwirklicht, wenn nicht nur die entsprechenden Skalen verwendet werden.

Aus der typischen Messung des Klimas über die subjektiven Klimabewertungen ergeben sich eine Reihe von methodischen und inhaltlichen Herausforderungen. Da das Klima (als mittlere Klimabewertung von Personen auf »Ebene 1«) als Merkmal einer Gruppe oder Einrichtung auf »Ebene 2« konzeptualisiert ist, folgen direkt fast sämtliche Probleme der Forschung in Mehrebenen-Strukturen. Ein scheinbar naheliegender Ausweg besteht darin, nur Merkmale auf Ebene 2 (Gruppen oder Abteilungen) zu betrachten. Entsprechend nutzen Auty \& Liebling (2019) zur Bestimmung der Rolle des Klimas (bzw. der »moral performance«) beispielsweise nur Daten auf Ebene der Einrichtungen, wobei die Autorinnen auf Daten von 224 Messun- 
gen auf Einrichtungsebene zurückgreifen können. Ein Risiko besteht jedoch in der voreiligen Übertragung der Effekte auf Aggregatebene auf die einzelnen Personen (ökologischer Fehlschluss): Zusammenhänge auf Makro-Ebene müssen sich nicht auf Mikro-Ebene bestätigen, besonders wenn die Übereinstimmung innerhalb von Einrichtungen gering ist (vgl. Nezlek, Schröder-Abé \& Schütz 2006).

In Deutschland sind vergleichbare Studien bisher nicht vorhanden und angesichts der föderalen Strukturen auch nicht ohne Weiteres zu erwarten. ${ }^{7}$ Ganz im Gegenteil beschreiben bisherige Studien meist wenige Einrichtungen und Abteilungen, oft sogar nur einzelne Abteilungen oder Justizvollzugsanstalten (siehe Abschnitt 2). Um über die deskriptive Auswertung hinaus wichtige Fragen der Ursachen und Wirkungen des Klimas zu beantworten, werden in einigen Veröffentlichungen Zusammenhänge ausschließlich auf Personenebene ausgewertet (Neubacher \& Boxberg 2018; Stasch et al. 2018; Suhling, Köhler \& Bernardi 2009; Wößner \& Schwedler 2014). Obwohl eine solche Perspektive wie schon dargestellt durchaus ihre Berechtigung hat, verleitet dieses Vorgehen leider dazu, die Klimawahrnehmung bzw. -bewertung mit Aussagen zum Klima zu vermischen. Deutlich wird diese Herausforderung beispielsweise im Aufsatz von Wößner \& Schwedler (2014, 872), die einerseits überwiegend konsistent von Klimawahrnehmung sprechen, allerdings in der Interpretation doch schlussfolgern, dass »[...] a positive prison climate was indeed [...] associated with positive treatment changes in dynamic risk factors « - gemessen und überprüft wurden lediglich Klimabewertungen auf Ebene 1.

Liegen Daten auf Personen- und Kontextebene vor, besteht eine Möglichkeit des Umgangs in der Aggregation (z. B. Mittelwert von Personenmerkmalen als Merkmale der Abteilung) oder Disaggregation (Zuweisung von identischen Kontextmerkmalen zu allen Personen innerhalb dieses Kontexts) von Daten. Ersteres führt zu einer Auswertung auf Ebene 2, letzteres zu einer Auswertung nur auf Ebene 1. Diese Varianten führen zu durchaus kreativen Analysestrategien. In einem aktuellen Beitrag (Sauter et al. 2019) werden beispielsweise in einem ersten Schritt Einschätzungen der Bediensteten pro Einrichtung gemittelt (Aggregation) und in einem zweiten Schritt diese Aggregate den einzelnen Inhaftierten der Abteilungen zugewiesen (Disaggregation). Als Basis für die Auswertungen werden dann allerdings die Inhaftierten verwendet $(\mathrm{N}=232)$, obwohl - wie Hox, Moerbeek \& van de Schoot $(2018,3)$ treffend formulieren - die "proper sample size for these varia-

7 Im Maßregelvollzug gibt es allerdings eine vergleichbar umfangreiche Untersuchung (Schalast 2019/Schalast \& Sieß 2018). bles is of course the number of higher-level units". Wird die Mehrebenenstruktur und damit die Abhängigkeit der Daten ignoriert, drohen nicht nur ein ökologischer Fehlschluss, sondern zugleich eine Überschätzung der effektiven Stichprobengröße und damit ein massiv erhöhtes Fehlerniveau (Hox et al. 2018).

Die deshalb eigentlich naheliegende und sinnvolle gemeinsame Modellierung beider Ebenen über Mehrebenenanalysen steht in der deutschsprachigen Klimaforschung vor allem vor der Herausforderung, dass einzelne Studien häufig nur eine geringe Zahl von Einrichtungen auf Ebene 2 berücksichtigen können. Selbst die großangelegte Studie des KFN (Baier et al. 2014/Klatt et al. 2017) umfasst "nur" 18 Einrichtungen, eine eigene Auswertung (Guéridon \& Strecker, im Druck) 11 Abteilungen. Da ein Ignorieren der Mehrebenenstruktur jedoch im Zweifel noch problematischer gewichtet werden kann, scheint einerseits gerade bei wenigen Abteilungen zumindest eine Kontrolle der Abteilung über die Verwendung von fixed-effects-Modellen angezeigt, andererseits scheinen Mehrebenenanalysen zumindest keinen zusätzlichen Schaden anzurichten (Gelman \& Hill 2007; Robson \& Pevalin 2016).

Dass Klimaforschung aber auch anders möglich ist, zeigen nicht nur die Strategie von Liebling \& Auty (2019), sondern vor allem die Projekte aus der Schweiz (Isenhardt et al. 2020) und den Niederlanden (van Ginneken et al. 2018; van Ginneken et al. 2019). Hervorzuheben sind zudem, wenn auch der Fokus auf dem Maßregelvollzug liegt, die Arbeiten von Schalast (2019).

\subsection{Unterschiede in der Klimabewertung zwischen und innerhalb von Gruppen}

Wären die beteiligten Personen (Bedienstete, Inhaftierte) in der Lage, das Klima in ihrer Einrichtung »objektiv« zu beschreiben, sollten abgesehen von Messfehlern alle beteiligten Personen identische Antworten geben. Dies ist aber offensichtlich nicht der Fall (vertiefend dazu Guéridon \& Strecker, im Druck; van Ginneken \& Nieuwbeerta 2020).

Das schon dargestellte Problem der Subjektivität von Klimawahrnehmungen und -bewertungen geht aber über die messmethodische Herausforderung hinaus. In der Tat zeigt die Mehrheit der Studien in Einklang mit der internationalen Forschung - wenn auch nicht durchgehend systematische Unterschiede in der Klimawahrnehmung zwischen Personen und Gruppen auch innerhalb von Einrichtungen (Guéridon \& Strecker, im Druck; Isenhardt et al. 2020; Pniewski, Elsner \& König 2019; Schalast 2016; Schalast \& Tonkin 2016). Dies ist zum Teil trivial, weil Be- 
dienstete beispielsweise natürlich nicht sämtliche Interaktionen zwischen den Inhaftierten beobachten können. Allerdings stellt sich die Frage, ob auch die aggregierten subjektiven Einschätzungen bestimmten »Urteilsfehlern« oder auch bestimmten explizit motivierten Verzerrungen unterliegen. Dabei ist es erst einmal unerheblich, ob nun Inhaftierte möglicherweise die Unterstützung durch die Bediensteten niedriger bewerten oder die Bediensteten diese zu hoch bewerten - jede Form von Unterschieden in der Bewertung wirft schlichtweg die Frage auf, ob ein einzelner Wert, möglicherweise auch nur von einer bestimmten Gruppe, repräsentativ für das Klima stehen kann. Entscheidet man sich für das Aggregieren der Daten verschiedener Gruppen, stellt sich die nächste Frage: Sollte nach Gruppengröße gewichtet werden (was den Inhaftierten ein höheres Gewicht gäbe) oder nicht (durch eine einfache Mittelung der Gruppenmittelwerte)? Sollte stattdessen doch besser ganz auf die Mittelung über Gruppen hinweg verzichtet werden, führt dies direkt zurück zur Frage, ob überhaupt ein (alternativer) einzelner Wert für das Klima einer Einrichtung bestimmt werden kann.

Da auch Studien aus dem deutschen Justiz- und Maßregelvollzugskontext systematische Unterschiede zwischen Bediensteten und Inhaftierten nahelegen (besonders auf der Dimension »Unterstützung durch Bedienstete«), scheint die Erhebung und Differenzierung dieser beiden Gruppen geboten (Guéridon \& Strecker, im Druck). Entsprechend hilfreich ist eine getrennte Normierung, wie sie im Manual zum EssenCES umgesetzt ist (Schalast \& Tonkin 2016).

\subsection{Wie stabil ist das Klima?}

Der überwiegende Teil der Literatur beschreibt das Klima als »stabil dynamisch «: Es verändert sich über die Zeit, der Prozess wird jedoch eher als langsam eingeschätzt. Dabei kann zwischen intraindividuellen Veränderungen der Klimabewertung (z.B. Anfang und Ende einer Behandlung) und kontextuellen Veränderungen des Klimas unterschieden werden (siehe vorheriger Abschnitt). In der Forschung zu Justizvollzugseinrichtungen wird die zeitliche Dimension bisher allerdings empirisch kaum berücksichtigt.

Individuelle Veränderungen der Klimawahrnehmung scheinen durchaus regelmäßig und auch systematisch zu sein (Ortmann 2002; Seifert \& Thyrolf 2010). Aus der Perspektive der Klimaforschung handelt es sich hierbei gegebenenfalls um eine problematische Verzerrung, wenn das Klima auf Ebene der Einrichtung verortet wird. In diesem Fall muss hinterfragt werden, ob es die individuellen Erfahrungen sind, die sich tatsächlich ändern, ob sich das
Klima der Einrichtung geändert hat oder ob es sich um methodische Artefakte handelt (z. B. »response shift«-Phänomene, für eine ausführlichere Diskussion siehe Guéridon \& Strecker, im Druck).

Wie schnell und unter welchen Bedingungen sich das Klima einer Abteilung oder Gruppe tatsächlich ändert, ist bisher allerdings nur selten untersucht worden. Dies mag auch daran liegen, dass Längsschnittstudien aufwendig sind, die beteiligten Personen belastet werden und schwer bestimmbar ist, inwieweit Veränderungen lediglich auf eine andere Gruppenzusammensetzung zurückzuführen sind. Positiv hervorzuheben sind einige Arbeiten, die zumindest für jährliche (Mohr et al. 2019) oder halbjährliche (Pniewski 2019/Pniewski, Elsner \& König 2019) Rhythmen durchaus Verschiebungen der mittleren Klimabewertung aufzeigen.

Wichtig scheint jedenfalls, das Klima nicht nur an einem Zeitpunkt zu messen, sondern im Verlauf. Ob eine solche Begleitforschung von externen Arbeitsgruppen aus eigenen Mitteln und/oder Projektgeldern geleistet werden kann, scheint fraglich. Insofern spricht einiges dafür, Klimaforschung auch von »innen« oder im Falle der Klimamessung auch ganz in Verantwortung der Einrichtungen $\mathrm{zu}$ betreiben (zu einem vergleichbaren Plädoyer im Bereich der Gewalt-Dunkelfeldforschung in Haft siehe Suhling \& Prätor, im Druck).

\subsection{Ursachen und Wirkungen des Klimas sind unklar}

Aus den bereits dargestellten Herausforderungen der Klimaforschung ergibt sich ein letzter, besonders schwerwiegender Umstand. Auch wenn die Zahl der internationalen Studien zu Ursachen und Wirkungen des Klimas durchaus umfangreich ist (vgl. Guéridon \& Suhling 2018), handelt es sich fast ausschließlich um Beobachtungsstudien mit kleinen Stichproben auf Ebene 2, die in der Regel nur Merkmale der Personen, nicht aber des Kontexts berücksichtigen. Die aus der Praxis oft formulierte Frage, wie denn »Klimawandel « aktiv gestaltet werden kann, welche organisatorischen, personellen, architektonischen, strategischen und gestalterischen Voraussetzungen dafür nötig oder hilfreich sind, können oft nur auf Grundlage theoretischer Überlegungen oder Analogien beantwortet werden. In Anlehnung an die Russo-Williamson-These (Illari \& Russo 2014) scheint gerade für die Bestimmung der kausalen Wirkung des Klimas neben überzeugenden Daten auch ein plausibler Wirkmechanismus notwendig. An dieser Stelle spielen alle bisher benannten Herausforderungen zusammen: die Definition des Konstrukts und die fehlende Aus- 
einandersetzung mit dessen Grenzen (wenn alles Teil des Klimas ist, kann das Klima nichts erklären!), die Mehrebenenstruktur und die verschiedenen Analyseebenen, die geringen Stichprobengrößen auf Ebene 2, die fehlende längsschnittliche Betrachtung, die fehlende Berücksichtigung von Kontextmerkmalen usw.

Gerade wenn das Klima als Kontextfaktor betrachtet wird, muss früher oder später die Frage beantwortet werden, wie denn eine solche (emergente) Eigenschaft genau wirken soll oder wozu das Klima überhaupt gemessen wird. Handelt es sich beim Klima vielleicht nur um ein pragmatisches, deskriptives globales Konstrukt zur Datenreduktion und Beschreibung oder um ein Epiphänomen ohne kausale Wirkung jenseits der individuellen Erlebnisse? Mit anderen Worten: Beeinflusst das Klima die Klimabewertung und all die vermuteten Faktoren oder ist das Klima nur eine globale Beschreibung ohne eigene Wirkung über seine konstituierenden Merkmale hinaus? Unabhängig von der konkreten Antwort auf diese Frage ist (pragmatische) Klimaforschung möglich und sinnvoll, allerdings ergibt sich unter anderem die Frage, wie genau Ergebnisse interpretiert werden sollten. Es scheint schlichtweg wenig vielversprechend, Veränderungen im Klima als Ursache zu betrachten, wenn die eigentlich relevante Ebene die Klimafacetten oder gar die direkten alltäglichen Interaktionen sind. Insofern kann nur erneut betont werden, wie wichtig die konzeptuelle Klarheit ist, welche Wirkung auf welcher Ebene vermutet wird und was entsprechend gemessen werden sollte.

Akzeptiert man vorerst die Prämisse, dass das Klima eine Wirkung haben kann, stellt sich umso mehr die Frage nach differenzieller Wirkung. Einige Theorien und Daten sprechen am ehesten dafür, dass gutes Klima negative Effekte abfedern kann, negatives Klima dagegen einen eigenständigen Stressor darstellt. Zum Beispiel entwickeln und prüfen Dollard \& Bakker (2010) in einem Aufsatz ein Modell des "psychosocial safety climate«, in dem ein direkter negativer Effekt auf Anforderungen, ein direkter positiver Effekt auf Ressourcen und moderierende Effekte auf psychologische Gesundheitsprobleme vermutet werden. Ein großer Teil der Klimaforschung im Justizvollzug kann aber wegen des querschnittlichen Designs und der konzeptuellen Unklarheiten gar nicht trennscharf zwischen Ursachen, Wirkungen, Korrelaten und logischen Implikationen unterscheiden, gerade wenn nur individuelle Klimabewertungen berücksichtigt werden. Schon theoretisch oder gar logisch bedingen sich das Klima und viele vermutete Wirkungen gegenseitig. Das Beispiel von Klimabewertung und Arbeitszufriedenheit kann hier erneut exemplarisch angeführt werden. Letztlich bleibt für viele Fragestellungen nur längsschnittliche Forschung zur
Bestimmung von Ursachen und Wirkungen eine echte Option. Experimentelle Manipulationen des Klimas selbst scheinen dagegen vorerst wenig vielversprechend, denn dazu müssten die relevanten Bedingungen ja bereits bekannt sein. ${ }^{8}$ Zugleich stellt sich die Frage, wie umfangreich sich Kontextfaktoren, die das Klima beeinflussen oder ausmachen, überhaupt verändern, denn ohne Veränderung lässt sich auch im Längsschnitt keine Kausalität beschreiben.

\section{Fazit}

Das Klima im Justizvollzug scheint auch in der deutschen Strafvollzugsforschung mehr in den Fokus zu rücken. Bisherige Untersuchungen betrachten häufig eher therapeutisch ausgerichtete Einrichtungen (Sozialtherapie, Sicherungsverwahrung, Jugendstrafvollzug), beschränken sich meist auf Querschnittuntersuchungen, verwenden eine Reihe verschiedener Instrumente und berücksichtigen immer wieder nur einzelne Gruppen (Inhaftierte oder Bedienstete). Zudem handelt es sich meist um »kleinere« Untersuchungen mit wenigen Einrichtungen oder Gruppen. Die Rücklaufquoten sind bei den Inhaftierten in der Regel sehr gut (> 80\%), bei den Bediensteten allerdings geringer. Die Projektübersicht zeigt deutlich, dass Klimaforschung möglich und vielversprechend ist. Die größte Lücke bisheriger Projekte besteht im Fehlen wiederholter Messungen des Klimas. Ergebnisse einiger kleinerer Projekte (Mohr et al. 2019; Pniewski 2019/Pniewski, Elsner \& König 2019) zeigen aber, dass auch längsschnittliche Betrachtungen möglich sind, wenn Durchführung und Auswertung als Routine aufgegriffen werden. Wichtig ist dabei, dass Bedienstete vor Ort auch ein Interesse am Klima und gegebenenfalls seiner Verbesserung haben. Dies betrifft nicht nur, aber in besonderem Maße die Leitung der Einrichtungen.

Eine große Herausforderung für die Klimaforschung im Justizvollzug scheint, die bestehenden Instrumente auch psychometrisch noch besser abzusichern und im Falle von (Neu-)Übersetzungen und Neuentwicklungen zumindest ein weiteres etabliertes Verfahren zu verwenden. Dabei muss natürlich die Frage der Ökonomie berücksichtigt werden, da einige Verfahren sehr umfangreich sind. Aus unserer Erfahrung bietet sich das EssenCES aufgrund

8 Dies gilt nicht gleichermaßen für experimentelle Manipulationen von möglichen Ursachen eines guten Klimas. 
seiner Sparsamkeit und Verbreitung an, ist jedoch nicht alternativlos. Es handelt sich aber bisher um das einzige Verfahren, das überhaupt Normwerte auf Einrichtungsebene zur Verfügung stellt, die mit weiterer Verwendung abgesichert und erweitert werden können. Die Normierung auch neuer Verfahren ist so oder so eine wichtige Aufgabe für die Klimaforschung. Dafür müssen aber großangelegte Studien durchgeführt werden, die nicht nur einzelne, sondern eine große Zahl von Einrichtungen berücksichtigen. Diese würden sich zudem auch für eine Untersuchung von Ursachen und Wirkungen des Klimas eignen, vor allem wenn Abteilungen mehrfach untersucht werden. Nur so können letztlich auch methodische und statistische Herausforderungen der Mehrebenenstruktur angemessen berücksichtigt werden.

Zugleich sollte die theoretische Ebene der Klimaforschung stärker berücksichtigt werden. Die hier angeführten Argumente verneinen nicht die Sinnhaftigkeit der Betrachtung von Zusammenhängen von Klimawahrnehmungen, -bewertungen und verschiedenen Kriterien, nur handelt es sich bei diesen Fragen in den meisten Fällen nicht um "Klimaforschung", sondern eher um Forschung zur »individuellen Lebensqualität inhaftierter Personen «. Wichtig scheint daher, im Falle der alleinigen Auswertung auf Personenebene auch konsistent von Klimawahrnehmung, Klimabewertung oder eben auch individueller Lebensqualität im Justizvollzug zu sprechen, aber eben nicht von »Klima«. Dieser Begriff sollte für das Konstrukt auf Gruppen-, Abteilungs- bzw. Einrichtungsebene reserviert bleiben.

Auch wenn im deutschen Justizvollzug bisher keine entsprechende Studie durchgeführt wurde, zeigen Erfahrungen aus der Schweiz (Isenhardt et al. 2020), den Niederlanden (van Ginneken et al. 2018), Großbritannien (Auty \& Liebling 2019) und eben auch dem deutschen Maßregelvollzug (Schalast 2019/Schalast \& Sieß 2018), dass auch solche Studien auf Aggregatebene möglich sind. Eine zentrale Aufgabe der Forschung im Justizvollzug besteht daher darin, nach Möglichkeit die Stichprobe auf Ebene 2 zu vergrößern, besonders wenn die Fragestellung konzeptuell eindeutig auf dieser Ebene angesiedelt ist. Alternativ sollte im Falle der Untersuchung mehrerer Einrichtungen zumindest die Abhängigkeit der Daten kontrolliert werden.

Wenn das Klima als wichtiger Faktor ernstgenommen werden soll und wirklich psychologisch informierte, aktiv gestaltete Umwelten angestrebt werden, braucht es auch ein entsprechendes Forschungsprogramm, um empirisch abgesicherte Faktoren und Strategien zu ermitteln. Dazu werden neben validen Messinstrumenten auch langfristig und großangelegte Forschungsprojekte benötigt, die ge- nügend Abteilungen wiederholt untersuchen, wenn möglich auch mit experimentellen Methoden.

Für die Praxis sowie die wichtige lokale Klimamessung und -forschung stellt sich dieses Problem weniger, wenn auf die Prüfung von Theorien zugunsten einer deskriptiven Betrachtung verzichtet wird. Da zumindest bisher keine umfangreichen Ergebnisse zur Reduzierbarkeit der Klimafacetten auf einzelne globale Indikatoren vorliegen und auch sonst viele konzeptuelle Fragen offen sind, lassen sich nur eingeschränkt Empfehlungen für diese angewandte Seite der Klimaforschung ableiten. Sinnvoll scheint aber, anhand von globalen Einschätzungen (»Wie ist das Klima zwischen Inhaftierten und Bediensteten?«) oder kurzen Instrumenten - wie dem EssenCES oder dem PGCI - das Klima in einer Art Monitoring zu erfassen (wie etwa bei Pniewski 2019/Pniewski, Elsner \& König 2019; Mohr et al. 2019) und die Messung nur im Falle deutlicher Veränderungen, auffälliger Werte sowie auch bei bestimmten Fragestellungen um eine intensive und differenzierte Analyse zu erweitern (mit MQPL, CKS, o.Ä.).

Dabei sollten grundsätzlich die Perspektiven von Bediensteten und Inhaftierten erfasst werden. Dies scheint auch über die wiederholt berichteten, systematischen Unterschiede zwischen den Gruppen hinaus auch eine bessere Grundlage für die gemeinsame Interpretation und Arbeit am Klima zu sein. Letztendlich sollte auch das ein wesentliches Anliegen der Klimaforschung im Justizvollzug sein.

\section{Literatur}

Adler, L. (2015). Das Haftklima im Jugendstrafvollzug und seine Abhängigkeit von strukturellen Determinanten der Inhaftierungssituation. Universität Erlangen-Nürnberg: Unveröffentlichte Masterarbeit.

Auty, K.M. \& Liebling, A. (2019). Exploring the relationship between prison social climate and reoffending. Justice Quarterly; https:// doi.org/10.1080/07418825.2018.1538421.

Baier, D. \& Bergmann, M.C. (2013). Gewalt im Strafvollzug - Ergebnisse einer Befragung in fünf Bundesländern. Forum Strafvollzug 62 (2), 76-82.

Baier, D., Pfeiffer, C. \& Bergmann, M.C. (2014). Beeinflussen Merkmale von Justizvollzugsanstalten das Gewaltverhalten der Gefangenen. In Neubacher, F. \& Kubink, M. (Hrsg.), Festschrift für Michael Walter (473-490). Berlin: Duncker \& Humblot.

Beijersbergen, K.A., Dirkzwager, A.J., van der Laan, P.H. \& Nieuwbeerta, P. (2016). A social building? Prison architecture and staff-prisoner relationships. Crime \& Delinquency 62 (7), 843-874.

Bosma, A.Q., van Ginneken, E.F.J.C., Sentse, M. \& Palmen, H. (2019). Examining Prisoner Misconduct: A Multilevel Test Using Personal Characteristics, Prison Climate, and Prison Environment. Crime \& Delinquency; https://doi.org/10.1177/0011128719877347. 
Boxberg, V., Fehrmann, S.E., Häufle, J., Neubacher, F. \& Schmidt, H. (2016). Gewalt als Anpassungsstrategie? Zum Umgang mit Belastungen im Jugendstrafvollzug. Monatsschrift für Kriminologie und Strafrechtsreform 99 (5), 428-449.

Braune, P., Klapprott, J., Linz, P., Lösel, F. \& Runkel, T. (1983). Psychologische Organisationsentwicklung im Strafvollzug. In Lösel, F. (Hrsg.), Kriminalpsychologie (228-238). Weinheim: Beltz.

Dahle, K.-P. \& Steller, M. (1990). Coping im Strafvollzug: Eine Untersuchung zu Haftfolgen bei Jugendlichen. Zeitschrift für experimentelle und angewandte Psychologie 37 (1), 31-51.

Dollard, M.F. \& Bakker, A.B. (2010). Psychosocial safety climate as a precursor to conducive work environments, psychological health problems, and employee engagement. Journal of Occupational and Organizational Psychology 83 (3), 579-599.

Drenkhahn, K. (2011). Anstaltsklima im Strafvollzug - Weiches Kuschelthema oder harter Erfolgsfaktor? GreifRecht 11, 25-31.

Gelman, A. \& Hill, J. (2007). Data analysis using regression and multilevel/hierarchical models. Cambridge: University Press.

Girisch, J. (2014). Plötzlich gesund! Vom Sinn und der Notwendigkeit, (auch) im Justizvollzug gesundheitsförderliche Strukturen zu implementieren. Dissertation Universität Jena.

Guéridon, M. (2019). Abteilungsklima in der niedersächsischen Sozialtherapie. Ergebnisse einer Befragung von Inhaftierten und Bediensteten aller sozialtherapeutischen Abteilungen in Niedersachsen und Bremen. Unveröffentlichter Forschungsbericht. Celle: Kriminologischer Dienst.

Guéridon, M. \& Suhling, S. (2018). Klima im Justizvollzug. In Maelicke, B. \& Suhling, S. (Hrsg.), Das Gefängnis auf dem Prüfstand. Wiesbaden: Springer.

Guéridon, M. \& Strecker, V.-K. (im Druck). Same but different? Die Bewertung des Klimas in sozialtherapeutischen Einrichtungen durch Bedienstete und Inhaftierte. In Veröffentlichungsband der Tagung der Kriminologischen Gesellschaft 2019 in Wien.

Greve, W. (2001). Traps and gaps in action explanation: Theoretical problems of a psychology of human action. Psychological Review 108 (2), 435.

Heynen, E.J.E., Helm, G.H.P. van der, Stams, G.J.J.M. \& Korebrits, A.M. (2014a). Anwendungsmöglichkeiten des deutschen »Prison Group Climate Instrument « (PGCl) zur Erfassung des Gruppenklimas in (Jugend-)Strafanstalten. Monatsschrift für Kriminologie und Strafrechtsreform 97 (3), 224-231.

Heynen, E.J.E., Helm, G.H.P. van der, Stams, G.J.J.M. \& Korebrits, A.M. (2014b). Measuring group climate in a German youth prison: A German validation of the prison group climate instrument. Journal of Forensic Psychology Practice 14 (1), 45-54.

Heynen, E.J.E., Helm, G.H.P. van der, Behrens, E. \& Korebrits, A.M. (2014c). Das Gruppenklima im deutschen Jugendstrafvollzug. Ein Sachstandsbericht zur aktuellen »Prison Group Climate» Forschung in Nordrhein-Westfalen. Bewährungshilfe 61 (4), 410-421.

Hox, J.J., Moerbeek, M. \& van de Schoot, R. (2010). Multilevel analysis: Techniques and applications. New York: Routledge.

Illari, P. \& Russo, F. (2014). Causality: Philosophical theory meets scientific practice. London: Oxford.

Isenhardt, A., Mangold, C. \& Hostettler, U. (2020). Das soziale Klima in Gefängnissen und Anstalten des Schweizer Straf- und Massnahmenvollzugs: Unterschiede in der Wahrnehmung von Personal und Gefangenen. NKrim - Neue Zeitschrift für Kriminologie und Kriminalpolitik 1 (1), 53-66.
Klatt, T., Suhling, S., Bergmann, M.C. \& Baier, D. (2017). Merkmale von Justizvollzugsanstalten als Einflussfaktoren von Gewalt und Drogenkonsum - Eine explorative Studie. Monatsschrift für Kriminologie und Strafrechtsreform 100 (4), 250-271.

Klein, R., Schmucker, M. \& Lösel, F. (2015). Evaluation der sozialtherapeutischen Abteilungen der JVA Neuburg-Herrenwörth: Evaluationskonzept \& erste Ergebnisse. In DVJJ e.V. (Hrsg.), Jugend ohne Rettungsschirm. Dokumentation des 29. Jugendgerichtstages 14.-17.09.2013 in Nürnberg (375-391). Mönchengladbach: Forum Verlag Godesberg $\mathrm{GmbH}$.

Lehmann, A., Greve, W. \& Ansorge, N. (2006). Justizvollzug als Profession: Herausforderung eines besonderen Tätigkeitsbereichs. Baden-Baden: Nomos.

Liebling, A., Hulley, S. \& Crewe, B. (2011). Conceptualising and Measuring the Quality of Prison Life. In Gadd, D., Karstedt, S. \& Messner, S. (eds.), The Sage Handbook of Criminological Research Methods. London: Sage

Lösel, F., Köferl, P. \& Weber, F. (1987). Meta-Evaluation der Sozialtherapie: qualitative und quantitative Analysen zur Behandlungsforschung in sozialtherapeutischen Anstalten des Justizvollzugs. Stuttgart: Enke.

Mohr, M., Ueberbach, L., van der Helm, P. \& Heynen, E. (2019). Gruppenklimaforschung im Justizvollzug. Ein Interventionsansatz zur Förderung der Behandlungsmotivation junger Inhaftierter und der Organisations- und Personalentwicklung in der JVA Iserlohn. Forum Strafvollzug 68 (1), 29-34.

Moos, R.H. (1997). Evaluating treatment environments: The quality of psychiatric and substance abuse programs. New Brunswick: Transaction Publishers.

Neubacher, F. \& Boxberg, V. (2018). Gewalt und Subkultur. In Maelicke, B. \& Suhling, S. (Hrsg.), Das Gefängnis auf dem Prüfstand (195-216). Wiesbaden: Springer.

Nezlek, J.B., Schröder-Abé, M. \& Schütz, A. (2006). Mehrebenenanalysen in der psychologischen Forschung. Psychologische Rundschau 57 (4), 213-223.

Obergfell-Fuchs, J. (2017). »Lebensqualität im Strafvollzug«. In H.-J. Kerner, J. Kinzig \& R. Wulf (Hrsg.), Kriminologie und Strafvollzug. Symposium am 19. März 2016 (71-84). Tübingen, TÜKRIM 39.

Ortmann, R. (2002). Sozialtherapie im Strafvollzug. Freiburg: edition iuscrim.

Parker, C.P., Baltes, B.B., Young, S.A., Huff, J.W., Altmann, R.A., Lacost, H.A. \& Roberts, J.E. (2003). Relationships between psychological climate perceptions and work outcomes: a meta-analytic review. Journal of Organizational Behavior 24 (4), 389-416.

Pniewski, B. (2019). Qualitätssicherung im Jugendmaßregelvollzug. Behandlungsevaluation für die praktische Arbeit. Forum Strafvollzug 68 (2), 119-124.

Pniewski, B., Elsner, K. \& König, A. (2019). Behandlungsevaluation im Jugendmaßregelvollzug. Forensische Psychiatrie, Psychologie, Kriminologie 13 (3), 282-291.

Pniewski, B. \& Walsh, M. (2018). Schwierigkeiten bei der Implementierung von Evaluationsforschung in der Praxis. In Walsh, M., Pniewski, B., Kober, M. \& Armborst, A. (Hrsg.), Evidenzorientierte Kriminalprävention in Deutschland (253-268). Wiesbaden: Springer VS.

Prätor, S. \& Suhling, S. (in Vorb.). Gewaltmonitor in der Jugendanstalt Hameln. Unveröffentlichter Forschungsbericht. Celle: Kriminologischer Dienst. 
Reisig, M. \& Mesko, G. (2009). Procedural justice, legitimacy, and prisoner misconduct. Psychology, Crime and Law 15 (1), 41-59.

Robson, K. \& Pevalin, D. (2016). Multilevel modeling in plain language. Sage.

Sauter, J., Stasch, J., Seewald, K., Hausam, J. \& Dahle, K.-P. (2019). Let's Work Together - Occupational Factors and Their Correlates to Prison Climate and Inmates' Attitudes Towards Treatment. Frontiers in Psychiatry 10; https://doi.org/10.3389/fpsyt.2019. 00781.

Schalast, N. (2016): Lessons Learned From Past Studies and Directions for Future Research. In Schalast, N. \& Tonkin, M. (eds.), The Essen Climate Evaluation Schema - EssenCES. A Manual and more. Göttingen: Hogrefe.

Schalast, N. (2019). Aspekte der sozialen Atmosphäre im Justiz- und Maßregelvollzug. ... und die Auswirkungen auf den Behandlungserfolg. Forum Strafvollzug 68 (2), 111-118.

Schalast, N. \& Groenewald, I. (2009). Ein Kurzfragebogen zur Einschätzung des sozialen Klimas im Strafvollzug - erste Befunde auf Abteilungen des Regelvollzugs und der Sozialtherapie. In R. Haller \& J.-M. Jehle (Hrsg.), Drogen - Sucht Kriminalität (329-352). Mönchengladbach: Forum Verlag Godesberg.

Schalast, N. \& Sieß, J. (2018). Zusammenhänge des Stationsklimas mit objektiven Rahmenbedingungen psychiatrischer Stationen. Psychiatrische Praxis 45 (5), 242-247.

Schalast, N. \& Redies, M. (2005). Das Stationsklima als Wirkfaktor der Behandlung - Entwicklung eines Beurteilungsbogens. In N. Saimeh (Hrsg.), Was wirkt? Prävention, Behandlung, Rehabilitation (244-255). Bonn: Psychiatrie-Verlag.

Schalast, N. \& Laan, J.M. (2017). Measuring social climate in German prisons using the Essen Climate Evaluation Schema. The Prison Journal 97 (2), 166-180.

Schalast, N. \& Tonkin, M. (eds.) (2016). The Essen Climate Evaluation Schema - EssenCES. A Manual and more. Göttingen: Hogrefe.

Seifert, S. \& Thyrolf, A. (2010). Das Klima im Strafvollzug. Eine Befragung von Gefangenen einer sozialtherapeutischen Einrichtung. Neue Kriminalpolitik 22 (1), 23-31.

Smedslund, J. (1984). What is necessarily true in psychology? Annals of Theoretical Psychology 2, 241-272.

Stasch, J., Sauter, J. \& Dahle, K.-P. (2017). Die neue Einrichtung zum Vollzug der Sicherungsverwahrung in Berlin - Behandlungsklima im Fokus. Forensische Psychiatrie, Psychologie, Kriminologie 11 (1), 13-21.

Stasch, J., Yoon, D., Sauter, J., Hausam, J. \& Dahle, K.-P. (2018). Prison climate and its role in reducing dynamic risk factors during offender treatment. International Journal of Offender Therapy and Comparative Criminology 62 (14), 4609-4621.
Strecker, V.-K. (2018). Klima im niedersächsischen Justizvollzug. Eine empirische Studie zum Vergleich der Einschätzungen von Bediensteten und Inhaftierten sozialtherapeutischer Einrichtungen. Universität Hildesheim: Unveröffentlichte Masterarbeit.

Suhling, S., Köhler, A. \& Bernardi, C. (2009). Soziales Klima, Behandlungsmotivation und Behandlungserfolg in Sozialtherapeutischen Einrichtungen des Justizvollzugs und im Maßregelvollzug. In N. Saimeh (Hrsg.), Motivation und Widerstand. Herausforderungen im Maßregelvollzug (211-228). Bonn: Psychiatrie-Verlag.

Suhling, S. \& Prätor, S. (im Druck). Gewalterfahrungen im Jugendstrafvollzug - Implementation und Ergebnisse von Dunkelfeldstudien »von innen«. Veröffentlichungsband der Tagung der Kriminologischen Gesellschaft 2019 in Wien.

Tonkin, M., Howells, K., Ferguson, E., Clark, A., Newberry, M. \& Schalast, N. (2012). Lost in translation? Psychometric properties and construct validity of the English Essen Climate Evaluation Schema (EssenCES) social climate questionnaire. Psychological Assessment 24 (3), 573-580.

van den Boogaart, H. (2014). Ein gutes Klima ist nicht alles - ohne gutes Klima ist alles nichts. Warum sprechen wir so wenig über die therapeutische Gemeinschaft? Forum Strafvollzug 63 (4), 224-227.

van der Helm, P., Stams, G.J. \& van der Laan, P. (2011). Measuring group climate in prison. The Prison Journal 91 (2), 158-176.

van Ginneken, E.F., Palmen, H., Bosma, A.Q., Nieuwbeerta, P. \& Berghuis, M.L. (2018). The Life in Custody Study: The quality of prison life in Dutch prison regimes. Journal of Criminological Research, Policy and Practice 4 (4), 253-268.

van Ginneken, E.F., Palmen, H., Bosma, A.Q. \& Sentse, M. (2019). Bearing the weight of imprisonment: The relationship between prison climate and well-being. Criminal Justice and Behavior 46 (10), 1385-1404.

van Ginneken, E.F. \& Nieuwbeerta, P. (2020). Climate consensus: A multilevel study testing assumptions about prison climate. Journal of Criminal Justice 69; https://doi.org/10.1016/j.jcrim jus.2020.101693.

Vries, M.D., Brazil, I.A., Helm, G.V., Verkes, R.J. \& Bulten, B.H. (2018). Ward Climate in a High-Secure Forensic Psychiatric Setting: Comparing Two Instruments. International Journal of Forensic Mental Health 17 (3), 247-255.

Wolter, D. \& Boxberg, V. (2016). The perception of imprisonment and its effect on inmate violence. In C. Reeves (ed.), Experiencing imprisonment: Research on the experience of living and working in Carceral institutions (156-175). London: Routledge.

Wößner, G. \& Schwedler, A. (2014). Correctional treatment of sexual and violent offenders: therapeutic change, prison climate, and recidivism. Criminal Justice and Behavior 41 (7), 862-879. 